Conference Proceedings Paper

\title{
Improving the Analytical Performance of Weak Aptamers: DNA Isothermal Amplification Approaches
}

\author{
Ramón Lorenzo-Gómez ${ }^{1,2, *}$, Rebeca Miranda-Castro ${ }^{1,2}$, Noemí de-los-Santos-Álvarez ${ }^{1,2}$ and \\ María Jesús Lobo-Castañón ${ }^{1,2}$ \\ 1 Departamento de Química Física y Analítica, Universidad de Oviedo. Av. Julián Clavería 8, 33006 Oviedo, \\ Spain; mirandarebeca@uniovi.es (R.M.-C.); santosnoemi@uniovi.es (N.d.-1.S.-Á.); \\ mjlc@uniovi.es (M.J. L.-C.) \\ 2 Instituto de Investigación Sanitaria del Principado de Asturias. Av. de Roma, 33011 Oviedo, Spain \\ * Correspondence: lorenzoramon@uniovi.es
}

Received: date; Accepted: date; Published: date

\begin{abstract}
Affinity characterization is an essential but time-consuming task to develop reliable aptamers for tumor biomarker detection and is not always thoroughly addressed. For neutrophil gelatinase-associated lipocalin (NGAL), a potential biomarker of pancreatic cancer, two DNA aptamers were described with very different affinity. Likewise, another pair of DNA aptamers was developed with very different affinity for alpha-fetoprotein (AFP), a biomarker of hepatocellular carcinoma. In this work, we estimated the dissociation constant of these aptamers by means of a direct assay on magnetic beads modified with biomarker and electrochemical detection on screenprinted carbon electrodes. In order to improve the performance of these aptamers, we proposed the isothermal amplification of the aptamers for both biomarkers by rolling circle amplification (RCA). In the case of AFP aptamers, we also tried terminal deoxynucleotidyl transferase (TdT), a templateindependent amplification. Both DNA amplifications improved the sensitivity and the apparent binding constants of the aptamers for the two cancer biomarkers. Nevertheless, this improvement depends on the true affinity of the binding pair, which ultimately limits their analytical usefulness.
\end{abstract}

Keywords: aptamer; dissociation constant; rolling circle amplification; terminal deoxynucleotidyl transferase; neutrophil gelatinase-associated lipocalin; alpha-fetoprotein

\section{Introduction}

Aptamers are synthetic receptors of nucleic acid nature that can bind to almost any type of target. These single-stranded DNA or RNA oligonucleotides are also known as "chemical antibodies" because they fold into unique 3D structures that allow them to interact with their cognate target in a similar way to the antibody-antigen interaction. The strength of the aptamer-target affinity binding is typically characterized by the equilibrium dissociation constant, $\mathrm{K}_{\mathrm{d}}$, which ultimately determines the analytical usefulness of the aptamer. The estimation of this parameter requires methodologies sensitive enough to quantify usual $K_{d}$ values within $\mathrm{pM}-\mathrm{nM}$ range. In this sense, aptamers can be directly coupled to DNA amplification techniques, transforming the aptamer-target recognition event into a nucleic acid amplification reaction [1]. Isothermal amplification schemes are especially relevant as, unlike PCR, they do not require the use of thermal cycles, thus being more eligible to miniaturization and implementation in decentralized point-of-care devices [2]. Among all isothermal DNA amplifications successfully applied to aptamer-based systems, rolling circle amplification (RCA) and terminal deoxynucleotidyl transferase based amplification (TdT) have turned out to be 
particularly convenient. Thus, RCA outstands due to its efficiency and amplification power [3], while TdT allows the extension of DNA sequences from their $3^{\prime}$ end in a template-independent process [4].

In this work, we have studied the affinity of two aptamers evolved against lipocalin 2 or neutrophil gelatinase-associated lipocalin (NGAL), potential biomarker of pancreatic cancer [5], and two aptamers raised against alpha-fetoprotein (AFP), biomarker of hepatocellular carcinoma [6,7], by means of heterogeneous direct binding assays with electrochemical detection. In addition, the possibility of enhancing the performance of these receptors by implementation of isothermal DNA amplification, either RCA or TdT, was evaluated.

\section{Materials and Methods}

\subsection{Reagents and Apparatus}

Detailed information is available in Refs [8,9].

\subsection{Experimental Protocols}

\subsubsection{Electrochemical Detection of RCA on Screen-Printed Gold Electrodes}

After the electrochemical conditioning, the Au working electrode was treated with a solution of the thiolated aptamer in phosphate buffer. To block the remaining bare Au sites, the surface was further treated with $p$-aminothiophenol. Then, the padlock hybridization and circularization were performed covering the working electrode with ligation mix (T4 DNA ligase and padlock in $1 \times$ ligase buffer), and then with RCA mix (phi29 DNA polymerase and dNTPs in $1 \times$ polymerase buffer). The amplification was carried out for $15 \mathrm{~min}$ at $37^{\circ} \mathrm{C}$. Subsequently, the electrode was firstly treated with a 6-FAM-labeled reporter probe and secondly with peroxidase-anti-fluorescein conjugate (antiFHRP). Finally, the substrates 3,3',5,5'-tetramethylbenzidine (TMB) and $\mathrm{H}_{2} \mathrm{O}_{2}$ were added to the cell, covering the three electrodes, and the enzymatic reaction proceeded for $30 \mathrm{~s}$. Chronoamperometry was carried out immediately after at $0 \mathrm{~V}$ for $60 \mathrm{~s}$.

\subsubsection{Electrophoretic Detection of TdT-Mediated Amplification Products}

TdT amplification was performed in solution, preparing reaction mixes with different concentrations of labeled dATP and incubating them for $1 \mathrm{~h}$ at $37^{\circ} \mathrm{C}$. Then, the solutions were treated with T40 probe. After enzyme deactivation, the solutions were cooled to favor the hybridization of the elongated aptamer with the T40 probe. Finally, the generated DNA duplexes were analyzed by electrophoresis in an agarose gel.

\subsubsection{Direct Assays on Biomarker-Modified Magnetic Beads}

Heterogeneous direct binding assays on NGAL or AFP-modified magnetic beads (MBs) were performed mixing a fixed amount of MBs with solutions of increasing concentrations of aptamers. In the case of binding assays without DNA amplification, the incubation with aptamers was directly followed by the enzymatic labeling step with antiF-HRP. For the electrochemical measurement, the MBs were placed on the working electrode of a carbon screen-printed cell (SPCE) and entrapped magnetically with the aid of a magnet. Finally, the SPCE was covered with substrates solution, and the enzymatic reaction and chronoamperometric measurement were carried out as mentioned above.

When RCA was used for signal enhancement, the binding step with anti-biomarker aptamers was followed by three extra steps: padlock hybridization and ligation, RCA, and reporter probe hybridization. Finally, the enzymatic labeling with antiF-HRP, enzymatic oxidation of TMB and chronoamperometric measurement were carried out as in the previous cases. For TdT amplification, the next step to the interaction with anti-AFP aptamers was the TdT amplification reaction, followed by the enzymatic labeling with a streptavidin-peroxidase conjugate (SA-HRP), the enzymatic oxidation of TMB and the chronoamperometric measurement. 


\section{Results and Discussion}

\subsection{RCA Optimization}

Rolling circle amplification relies on the high processivity of certain DNA and RNA polymerases to copy multiple times a circular single-stranded DNA template, previously hybridized to a short DNA or RNA primer. Thus, the resulting product is a long single-stranded DNA or RNA containing multiple tandem repeats of the complementary sequence to the original circular template, and can be electrochemically detected by hybridization with labeled reporter probes or by reaction with electroactive compounds [2].

We designed a RCA protocol using one of the anti-NGAL aptamer thiolated in its $5^{\prime}$ end for chemisorption onto gold surfaces. The amplification protocol comprised the following steps: (1) hybridization of a circularizable single-stranded DNA template (padlock) to the $3^{\prime}$ end of the aptamer, which served as the primer; (2) in parallel. Optimization of reagent concentrations (padlock, T4 DNA ligase, phi29 DNA polymerase, detection probe) and reaction times (padlock hybridization and ligation, RCA, detection probe hybridization) was carried out, increasing both the quickness and cost-effectiveness of the assay [8].

\subsection{TdT Optimization}

In addition to its unique ability to extend the $3^{\prime}$ end of a ssDNA without the need for template, TdT can incorporate not only the four naturally occurring nucleotides, but also synthetic modified analogues in a tunable ratio [4]. Thus motivated, we used biotinylated dATP mixed with unlabeled nucleotides to enable the enzymatic labeling of the elongated aptamer with streptavidin-peroxidase. First, we studied the biotin-dATP/dATP ratio to obtain the longest tail with a proper number of labels in solution. The elongated aptamers were analyzed by gel electrophoresis, and it was revealed that higher concentrations of labeled nucleotide led to shorter products [9].

TdT was then tested in a direct assay with electrochemical detection, using AFP-modified MBs and the aptamer. After the affinity interaction, the aptamer bound to the AFP-MBs was elongated with different ratios of biotin-dATP/dATP and then enzymatic labeling was performed with SA-HRP. In this case, higher concentrations of biotin-dATP, in the range 25-100 $\mu \mathrm{M}$, led to higher electrochemical signals, due to incorporation of a higher number of enzyme molecules per DNA strand. To strike a balance between enhancing the reaction rate (more unlabeled nucleotides) and the electrochemical signal (more labeled nucleotides), a concentration of $25 \mu \mathrm{M}$ of biotin-dATP was chosen as optimum. Since the rate of elongation is nucleotide dependent, we also studied the effect of using only dATP or an equimolar mixture of the 4 deoxyribonucleotides (dNTPs), and found that the mixture produced higher specific currents [9].

\subsection{DNA Amplified Direct Recognition of Cancer Biomarkers}

Once optimized, RCA was used to enhance the performance of both anti-NGAL and anti-AFP aptamers. Truncated versions of these aptamers were adapted to the RCA scheme extending their $3^{\prime}$ end with an adequate primer complementary to the padlock, separated from the main sequence with a 10-thymine spacer to ensure that the affinity reaction did not hinder the padlock hybridization. These aptamers were challenged to the recognition of their corresponding target biomarkers anchored on magnetic beads. An assay with no DNA amplification was performed in parallel, using the aptamers tagged with fluorescein. Binding curves for each system were obtained confronting a fixed amount of biomarker immobilized on MBs versus increasing concentrations of aptamer in solution.

The truncated anti-NGAL aptamer showed an apparent dissociation constant of $10 \pm 5 \mathrm{nM}$ with no amplification, while RCA pushed down this parameter to the picomolar range $(14 \pm 3 \mathrm{pM})$, thus producing an improvement of three orders of magnitude in the detectability of the target. However, both values were above the previously described $\mathrm{K}_{\mathrm{d}}(2.24 \mathrm{pM})$ [5]. Further binding measurements 
with other techniques (surface plasmon resonance spectroscopy and microscale thermophoresis) showed that the previously reported affinity of this aptamer might be overestimated [8].

In the case of AFP, the affinity of two different aptamers, AFP-S and AFP-L, was studied. AFP$\mathrm{S}$ was adapted to the RCA scheme, as previously mentioned, and this amplification produced a 6fold improvement in the apparent $\mathrm{K}_{\mathrm{d}}(188 \pm 33 \mathrm{nM})$ in comparison with the non-RCA amplified assay $(713 \pm 63 \mathrm{nM})$ [9]. These affinity values were close to those previously reported $(500 \mathrm{nM})$ [7], but the improvement attained with the amplification was much modest than the one achieved with the antiNGAL aptamer. On the other hand, AFP-L was amplified by TdT under optimized conditions. In this case, the TdT-amplified assay led to a 16-fold enhancement of the apparent $\mathrm{K}_{\mathrm{d}}(32 \pm 11 \mathrm{nM})$ compared to the non-amplified assay $(526 \pm 101 \mathrm{nM})$ [9]. These apparent $K_{d}$ values were above the previously reported one $(2.37 \mathrm{nM})[6]$, indicating that the "real" affinity of this aptamer is weaker than expected.

Funding: This research was funded by the Spanish Government (project RTI-2018-095756-B-I00) and Principado de Asturias Government (IDI2018-000217), co-financed by FEDER funds. R.L.-G. thanks the Spanish Government for a PhD fellowship (FPU16/05670).

Acknowledgments: The Unit of Biomedical and Biotechnological Tests of the Scientific and Technological Resources from Universidad de Oviedo is gratefully acknowledged.

Conflicts of Interest: The authors declare no conflict of interest.

\section{References}

1. Li, F.; Zhang, H.; Wang, Z.; Newbigging, A.M.; Reid, M.S.; Li, X.F.; Le, X.C. Aptamers facilitating amplified detection of biomolecules. Anal. Chem. 2015, 87, 274-292, doi:10.1021/ac5037236.

2. Lorenzo-Gómez, R.; Miranda-Castro, R.; de-los-Santos-Álvarez, N.; Lobo-Castañón, M.J. Electrochemical aptamer-based assays coupled to isothermal nucleic acid amplification techniques: New tools for cancer diagnosis. Curr. Opin. Electrochem. 2019, 14, 32-43, doi:10.1016/j.coelec.2018.11.008.

3. Feng, C.; Mao, X.; Yang, Y.; Zhu, X.; Yin, Y.; Li, G. Rolling circle amplification in electrochemical biosensor with biomedical applications. J. Electroanal. Chem. 2016, 781, 223-232, doi:10.1016/j.jelechem.2016.07.008.

4. Motea, E.A.; Berdis, A.J. Terminal deoxynucleotidyl transferase: The story of a misguided DNA polymerase. Biochim. Biophys. Acta Proteins Proteom. 2010, 1804, 1151-1166, doi:10.1016/j.bbapap.2009.06.030.

5. Lee, K.A.; Ahn, J.Y.; Lee, S.H.; Singh Sekhon, S.; Kim, D.G.; Min, J.; Kim, Y.H. Aptamer-based sandwich assay and its clinical outlooks for detecting lipocalin-2 in hepatocellular carcinoma (HCC). Sci. Rep. 2015, 5, 10897, doi:10.1038/srep10897.

6. Huang, C.J.; Lin, H.I.; Shiesh, S.C.; Lee, G.B. An integrated microfluidic system for rapid screening of alphafetoprotein-specific aptamers. Biosens. Bioelectron. 2012, 35, 50-55, doi:10.1016/j.bios.2012.02.024.

7. Dong, L.; Tan, Q.; Ye, W.; Liu, D.; Chen, H.; Hu, H.; Wen, D.; Liu, Y.; Cao, Y.; Kang, J.; et al. Screening and identifying a novel ssDNA aptamer against alpha-fetoprotein using CE-SELEX. Sci. Rep. 2015, 5, 15552, doi:10.1038/srep15552.

8. Lorenzo-Gómez, R.; Fernández-Alonso, N.; Miranda-Castro, R.; de-los-Santos-Álvarez, N.; Lobo-Castañón, M.J. Unravelling the lipocalin 2 interaction with aptamers: May rolling circle amplification improve their functional affinity? Talanta 2019, 197, 406-412, doi:10.1016/j.talanta.2019.01.057.

9. Lorenzo-Gómez, R.; González-Robles, D.; Miranda-Castro, R.; de-los-Santos-Álvarez, N.; Lobo-Castañón, M.J. On the electrochemical detection of alpha-fetoprotein using aptamers: DNA isothermal amplification strategies to improve the performance of weak aptamers. Biosensors 2020, 10, 46, doi:10.3390/bios10050046.

(C) 2020 by the authors. Submitted for possible open access publication under the terms and conditions of the Creative Commons Attribution (CC BY) license (http://creativecommons.org/licenses/by/4.0/). 